ISSN 1794-9831

E-ISSN 2322-7028

Vol. 14 No. 1

Ene - Jun 2017

Cúcuta, Colombia

Recibido:

24 de Mayo de

2016

Aprobado:

13 de Noviembre de 2016

* Enfermera

Magister en

Educación,

Especialista en

Salud Ocupacional.

Especialista Auditoria

en Gerencia de

Servicios de Salud.

Docente. Universidad

de los Llanos.

Villavicencio,

Colombia. Correo

electrónico: priscila.

pena@unillanos.

edu.co

** Médico.

Magister en

Gerontología. Médico

Internista. Hospital

Departamental

de Villavicencio.

Villavicencio,

Colombia Correo:

mavi99mes@hotmail.

com

*** Enfermera.

Doctor en Salud

Pública, Magister

en Salud Pública.

Docente de Cátedra.

Universidad de Los

Llanos. Villavicencio,

Colombia. Correo

electrónico: acastro@

unillanos.edu.co

**** Descriptores

en Ciencias de la

Salud (DeCS), en la

página http://decs.

bvs.br/E/homepagee.

htm de la Biblioteca

Virtual en Salud del

proyecto BIREME,

de la Organización

Mundial de la Salud

y de la Organización

Panamericana de la

Salud

\section{Caracterización, riesgos ocupacionales y percepción de salud de vendedores informales de lotería y chance ch $^{\dagger}$}

\author{
Amalia Priscila Peña-Pita* \\ Martha Cecilia Sarmiento-Mejía** \\ Ana Teresa Castro-Torres ${ }^{* * *}$
}

\title{
RESUMEN
}

Objetivo: analizar las características sociodemográficas, percepción de la salud y riesgos ocupacionales de la población de vendedores informales de lotería y chance de Villavicencio y Yopal. Materiales y Métodos: estudio descriptivo, transversal y prospectivo. La muestra no aleatoria estuvo integrada por 249 vendedores informales. Las variables estudiadas fueron: características sociodemográficas y económicas, afiliación al sistema general de salud, nivel de ingreso, responsabilidad en el hogar, propiedad de la vivienda, factores de riesgo ocupacionales y condiciones laborales. Como instrumento de recolección de información se utilizó la Encuesta del Ministerio de Salud para Trabajadores Informales. Resultados: la mayoría de vendedores de lotería y chance son mayores de 40 años, mujeres, casados o en unión libre. Se observó una baja afiliación a pensiones y riesgos laborales, y con ingresos inferiores a un salario mínimo legal mensual vigente en un $80 \%$. Las condiciones de trabajo, sobre todo las ambientales y de seguridad, son precarias; aun así, la auto percepción de salud como mala o regular no supera el 50\%. Conclusión: población muy vulnerable en cuanto a sus ingresos y condiciones futuras de ingreso para protección en la vejez.

PALABRAS CLAVE: condiciones de trabajo, salud laboral, riegos laborales ${ }^{* * * *}$.

\section{Para citar este artículo / Para citar este artigo/ To reference this article /}

Peña-Pita AP, Sarmiento-Mejía MC, Castro-Torres AT. Caracterización, riesgos ocupacionales y percepción de salud de vendedores informales de lotería y chance. Rev. cienc. cuidad. 2017; 14(1): 60-78.

† El presente estudio no se habría podido llevar a cabo sin la financiación de la Universidad de los Llanos y el apoyo de las Secretarías de Salud de Villavicencio y de Casanare. 


\section{Characterization, occupational risks and perception of health of informal lottery vendors}

ISSN 1794-9831

E-ISSN 2322-7028

Vol. 14 No. 1

Ene - Jun 2017

Cúcuta, Colombia

\section{ABSTRACT}

Objective: To analyze the socio-demographic characteristics, health perception and occupational risks of the population of street lottery retailers in Villavicencio and Yopal. Materials and Methods: Descriptive, cross-sectional, and prospective study. The non-aleatory sample was made by 249 informal vendors. The variables studied were: socio-demographic and economic characteristics, affiliation to the general health system, level of income, home responsibility, homeownership, occupational risk factors and work conditions. As an instrument of data collection the Department of Health for Informal Workers Survey was used. Results: most of the lottery retailers are older than 40, women, married or cohabiting. A low affiliation to pension and occupational risks was observed, and with incomes lower than a monthly minimum wage valid in an $80 \%$. The work conditions, most importantly the environment and security conditions, are precarious; nonetheless, the self-perception of health as bad or regular does not reach $50 \%$. Conclusion: very vulnerable population regarding income and future conditions of income for old age protection.

KEYWORDS: working conditions, occupational health, occupational risks. 
ISSN 1794-9831

E-ISSN 2322-7028

Vol. 14 No. 1

Ene - Jun 2017

Cúcuta, Colombia

\section{Caracterização, riscos ocupacionais e percepção de saúde de vendedores informais de loteria}

\section{RESUMO}

Objetivo: analisar as características sócio-demográficas, percepção da saúde e riscos ocupacionais da população de vendedores ambulantes de loteria nas cidades de Villavicencio e Yopal na Colômbia. Materiais e Métodos: estudo descritivo, transversal e prospectivo. A amostragem não aleatória esteve integrada por 249 vendedores informais. As variáveis estudadas foram: características sóciodemográficas e económicas, afiliação à rede de saúde, nível de ingresso, responsabilidade no lar, casa própria ou não, fatores de risco ocupacionais e condições de trabalho. Como instrumento de recolecção de informação se utilizou a Pesquisa de Opinião do Ministério de Saúde para Trabalhadores Informais. Resultados: a maioria de vendedores de loteria são maiores de 40 anos, mulheres, casadas ou em união libre. Observou-se uma baixa afiliação a Fundo Social de Pensão e Riscos de Trabalho, e com ingressos inferiores a um salário mínimo legal mensal vigente num $80 \%$. As condições de trabalho, sobre todo as ambientais e de seguridade, são precárias; ainda assim, a auto percepção de saúde como má ou regular não supera o $50 \%$. Conclusão: população muito vulnerável em quanto a seus ingressos e condições futuras de ingresso para proteção na velhice.

PALAVRAS-CHAVE: condições de trabalho, saúde do trabalhador, riscos ocupacionais. 


\section{INTRODUCCIÓN}

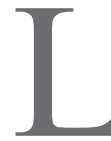

a economía informal no se puede ver como un fenómeno exclusivo de los países en vías de desarrollo. La globalización de la economía, hoy en día, ha evidenciado que la práctica de producir riqueza y crear empleo, fuera del marco legal, es también un fenómeno del mundo desarrollado en donde se es informal por decisión autónoma del trabajador, según la teoría institucionalista ${ }^{\dagger}$, o de acuerdo con la teoría estructuralista ${ }^{i \dagger}$, como consecuencia de un fenómeno que caracteriza a sectores de la estructura productiva y de empleo en los países en desarrollo, en donde evitar obligaciones de seguridad social les permite seguir en el mercado (1).

Los vendedores de lotería y chance hacen parte de los trabajadores informales en Colombia, considerados por el Departamento Administrativo Nacional de Estadística (DANE) como aquellos en los que "su relación de trabajo, de derecho o de hecho, no está sujeta a la legislación laboral nacional, al impuesto sobre la renta, a la protección social o a determinadas prestaciones relacionadas con el empleo (preaviso al despido, indemnización por despido, vacaciones anuales pagadas o licencia pagada por enfermedad" (2). La informalidad es la fuente de ingresos para la población de escasos recursos, constituye el 48,6\% de ocupados en Colombia para el año 2016 (3) y está emparentada con la pobreza y la desigualdad: del $20 \%$ de la población con menos ingresos en América Latina, un $73,4 \%$ está en situación de informalidad (4).

Galvis (5) afirma que, en Colombia, existen altos niveles de informalidad en las ciudades por fuera de Bogotá, Cali, Medellín y Bucaramanga, las cuales presentan mayor actividad económica. También se observa baja capacidad institucional de generar empleo y, en general, más mujeres se emplean en el sector informal, en detrimento de las condiciones laborales y expuestas a riesgos ambientales peligrosos, precario acceso a seguridad social (afiliación a salud, pensiones y riesgos), razones que ubican al trabajador informal dentro de la población vulnerable expuesta a la exclusión, siendo uno de los determinantes de la falta de cohesión social en amplios sectores de la población (6).

\footnotetext{
${ }^{\dagger}$ Quienes están en el sector informal lo hacen por decisión y no porque les toca hacerlo (1).

tit Explica la informalidad laboral como una alternativa de subsistencia, por defecto del crecimiento económico del país (1).
}

La informalidad laboral en los departamentos de Casanare y Meta, zona de influencia de este trabajo, se enfoca desde la teoría estructuralista lógica que también atribuye la informalidad a la supervivencia (7) y al desbalance entre la demanda y la oferta en el mercado de trabajo en un país en donde el tamaño de los sectores económicos depende de la diversificación de los niveles de la economía y la integración tecnológica (8).

De otra parte, la informalidad se puede asociar con menos ingresos para los hogares $\mathrm{y}$, según cálculos realizados con base en la Gran Encuesta Integrada de Hogares (GEIH), seis de cada diez ocupados en el área urbana pertenecen al sector informal (2), lo que afecta el bienestar general de dicha población. Frente a esta situación, en el país se han diseñado estrategias para aumentar la formalización y calidad del empleo (9) dentro de las cuales se destacan: esquemas tributarios simplificados para reducir la complejidad del sistema, automatizar y virtualizar los trámites y establecimiento de mecanismos de inspección, vigilancia y control empresarial; pero aún persiste la tercerización laboral (cooperativas) que contribuyen al detrimento del salario de los trabajadores.

Ahora bien, así como mediante el trabajo las personas pueden adquirir elementos esenciales para conservar un buen estado de salud, también se debe asegurar que las condiciones en las que se lleva a cabo sean dignas y seguras. La población de vendedores ambulantes -en este caso, los loteros y chanceros- está expuesta a factores ambientales tales como el daño solar, que es acumulativo e irreversible, genera fotoenvejecimiento, fotoder-matosis y fotocarcinogénesis, entre otros efectos (10) y, sin ser menos importantes, calambres y agotamiento o insolación por calor, sobre todo en personas mayores (11), el ambiente social en donde existen redes de control social y otras formas de acción colectiva (12) se constituye también en fuente de conflicto. Por lo tanto, limitar al máximo los riesgos laborales, mediante programas de prevención y apoyo social, se torna en una necesidad sentida y, en este contexto, el Ministerio del Trabajo (13) estableció un programa orientado a ayudar a las mujeres que son trabajadoras informales y jefes de hogar para que generen microempresas y obtengan ingresos marginales a partir de la creación de empresas rentables.

Aquí es importante mencionar la autopercepción de la salud (APES), que es un concepto que involucra elementos tanto subjetivos como objetivos, esto es, 
ISSN 1794-9831

E-ISSN 2322-7028

Vol. 14 No. 1

Ene - Jun 2017

Cúcuta, Colombia implica dimensiones provenientes desde lo biológico, el estado mental, la condición funcional, social y espiritual, y constituye un indicador que refleja las necesidades de atención de un colectivo poblacional para el sistema de salud, además de ser un predictor de mortalidad (14). Representa la percepción que tiene el individuo, de forma global, sobre diferentes dimensiones de su estado de salud que, si bien no proporciona información exacta de la misma, sí es un indicador indirecto de ella y está relacionado con el estilo de vida, la presencia de discapacidades o enfermedades (15) y resulta de utilidad para el sistema de salud, puesto que implica demanda de servicios.

Dada su repercusión, tanto en la economía del país como en la calidad de vida de los trabajadores, la informalidad ha sido motivo de diferentes análisis y estudios en el ámbito internacional y nacional. A nivel internacional se encuentra el de Guadarrama et al. (16), para el caso mexicano, en donde el análisis es especialmente relevante debido a que en la actualidad el porcentaje de informalidad muestra diversidad entre ciudades y va desde $52 \%$ en Oaxaca y $37,5 \%$ en Puebla y al 22,5\% en el Distrito Federal.

En Argentina, según la Organización Internacional del Trabajo (OIT) (17), uno de cada cuatro trabajadores es informal, con empleos de mala calidad, salarios bajos, largas jornadas de trabajo, falta de acceso a oportunidades de capacitación, dificultades para acceder al sistema judicial y al sistema de protección social -incluyendo la protección para la seguridad y salud en el trabajo-, fenómeno que dio origen al Programa de Trabajo Decente por País 2012-2015. Cassirer y Addati (18), en Guatemala, afirman que las responsabilidades familiares son importantes porque orientan a la mujer hacia el empleo informal y porque restringen sus actividades remuneradas al trabajar, es decir, limitan el tipo de actividades y la cantidad de tiempo que pueden dedicar a las actividades comerciales remuneradas.

En varios estudios descriptivos y transversales recientes (19-24), realizados en Colombia sobre el tema, se caracteriza y analiza la informalidad laboral desde aspectos relacionados con la probabilidad de ser informal o subempleado y las barreras para la formalización, hasta aspectos económicos como el efecto de las rigideces del mercado laboral sobre la informalidad, que evidencian que el incremento en los costos no salariales y en el salario mínimo tiene un efecto positivo sobre el tamaño del sector informal, así como en la probabilidad de transición de trabajadores desde el sector formal hacia el informal.

Estudios similares a esta investigación sobre trabajadores informales se han llevado a cabo en otras ciudades de Colombia, tales como los realizados en Bucaramanga (25), en Cauca por Muñoz y Chois (26) y en Villavicencio por parte de la Secretaría de Salud Municipal (27), además de los realizados por la Gobernación del Quindío (28) en población afro -también vulnerable-, en donde el 92,1\% se puede considerar como trabajador informal, bajo el indicador de afiliación al régimen contributivo. También están Salinas et al. (29) y la Universidad de Nariño (30), que abordaron características sociodemográficas y condiciones laborales similares a las planteadas en el presente trabajo: en su mayoría, la población tiene niveles bajos de escolaridad, percibe ingresos inferiores a un salario mínimo legal vigente, son cabeza de familia y tienen a su cargo entre 1 y 4 personas, lo que afecta su calidad de vida.

La importancia de investigaciones específicas acerca de la condiciones de los loteros y chanceros radica en que son ellos, con su trabajo, quienes contribuyen al crecimiento de las empresas de juegos de azar en Colombia, principal contribuyente del sistema de salud. Por lo tanto, merecen mayor respaldo, reflejado en garantías laborales como el pago de seguridad social y devengar un sueldo fijo.

Los resultados del presente estudio, al carecer de precedentes dentro del ámbito regional y nacional, aportan a la comprensión del fenómeno de la informalidad en la región y brindan información útil para su caracterización. Además, podrán ser tomados como base para el diseño de programas específicos de promoción y prevención aplicables a esta población vulnerable, dirigidos por las correspondientes Secretarías de Salud de Villavicencio y de Yopal.

\section{MATERIALES Y MÉTODOS}

Se realizó un estudio descriptivo, transversal y prospectivo, con enfoque cuantitativo. En Villavicencio, la población estuvo constituida por los 250 vendedores ambulantes de lotería agrupados 
por la beneficencia del departamento del Meta y 390 vendedores de chance; en Yopal correspondió a 53 vendedores de lotería y 160 vendedores de chance. El tamaño de la muestra $(n)$ se estableció mediante la función Statcalc del programa Epi-Info versión para Windows 7 (7.1.3), considerando los siguientes parámetros: $853(\mathrm{~N})$ total poblacional de vendedores y chanceros, un nivel de confianza $(Z)$ de $95 \%$ y un margen de error (E) de $5 \%$, peor resultado esperado $56 \%$, dando como resultado un $n$ de 260 vendedores. Sej tuvo en cuenta un $20 \%$ de rechazo, quedando así una muestra definitiva de 312 vendedores.

Sin embargo, debido a la sobreestimación de las unidades de análisis en Yopal, se tomaron todos -únicamente eran 64 vendedores de lotería y chance-, y en Villavicencio, en donde se tuvo que acudir al muestreo no probabilístico debido a la renuencia de algunos vendedores a participar en el estudio, la muestra quedó reducida a 185. De esta manera, los resultados se presentan sobre un total de 249 loteros y chanceros, que corresponden al 79,8\% de la muestra, porcentaje que representa la confiabilidad y validez alcanzada al final.

Para obtener datos sobre la población objeto de estudio se contó con la información de las empresas de lotería y chance, se aprovechó un evento planeado por una entidad administradora de cesantías en Colombia y se esperó en puntos de entrega de la lotería para invitar a participar a los loteros y guiarlos al sitio de recolección de la información. También se aplicó la encuesta en el sitio de trabajo.

El trabajador en informalidad se asume como aquel a quien no le son reconocidos: la contribución a salud, pensión y riesgos laborales, y percibe sólo pago de porcentaje sobre ventas, sin salario básico. Los criterios de inclusión fueron: ser vendedor de lotería o de chance, aceptar participar en el estudio a través del consentimiento informado, desarrollar su labor en el municipio de Villavicencio o Yopal. Los criterios de exclusión fueron: ser vendedor de otros objetos diferentes a lotería y chance, no aceptar participar en el estudio o pertenecer a municipios diferentes a Villavicencio o Yopal.

Las técnicas empleadas para la recolección de información fueron la observación no participante, centrada en detectar riesgos ocupacionales en los puestos de trabajo, y la Encuesta Nacional de Condiciones de Salud y Trabajo del Sector del Comercio Informal (31). Esta encuesta es un autoreporte y contiene información básica del trabajador: económica, de su área laboral, su seguridad social y sus condiciones de trabajo; en total la conforman 70 preguntas y se asumió el instructivo para el diligenciamiento empleado por los participantes en el estudio nacional, además de hacer las preguntas al vendedor. El instrumento es un consolidado de los departamentos que participaron en el estudio, de los cuales se llevó a cabo una estandarización de variables por parte de la Universidad de Antioquia, responsable del contrato ante el Ministerio del Trabajo. El uso del instrumento, publicado por el Ministerio de Salud en su página web, fue autorizado mediante comunicación telefónica con dicha entidad.

La encuesta está estructurada con preguntas cerradas que estudian las siguientes variables: caracterización sociodemográfica y económica (edad, género, procedencia, nivel educativo), afiliación al sistema general de salud (salud, pensión y riesgos laborales), nivel de ingreso (salario), responsabilidad en el hogar, estado civil y propiedad de la vivienda; factores de riesgo ocupacionales (físicos, de seguridad, ergonómicos) y también se indagaron las condiciones laborales (jornada, horas diarias de trabajo, días laborados en la semana, posición ocupacional, tipo de vendedor, forma de vender, tiempo en el oficio, trabajo adicional, porte de maletas). Las variables se clasificaron como cuantitativas (contínuas o discretas) y cualitativas, empleando escalas de respuesta dicotómicas y politómicas.

La Autopercepción de la Salud (APES) se obtuvo con base en la pregunta 40 de la Encuesta Nacional de Condiciones de Salud y Trabajo del Sector del Comercio Informal, eliminando la opción de excelente y quedando formulada así la pregunta: ¿Diría Usted, en general, que su salud es: muy buena, buena, regular o mala?.

En el estudio, el concepto de unidades de trabajo informal (UTI) se asumió de acuerdo a lo planteado por la Organización Panamericana de la Salud (OPS) y la Organización Mundial de la Salud (OMS) (32), donde se menciona que es "el conjunto de actividades e intervenciones intersectoriales de carácter
ISSN 1794-9831

E-ISSN 2322-7028

Vol. 14 No. 1

Ene - Jun 2017

Cúcuta, Colombia 
ISSN 1794-9831

E-ISSN 2322-7028

Vol. 14 No. 1

Ene - Jun 2017

Cúcuta, Colombia básicamente promocional, preventivo y participativo que se desarrollan para ofrecer a las personas protección frente a las amenazas para su salud y, a la vez, auspiciar la adquisición de conocimientos y capacidades para reconocer los riesgos y actuar oportunamente frente a ellos", que aseguran acciones concretas y contribuyen al mejoramiento de la calidad de vida de la población de vendedores de lotería y chance.

La recolección de información fue realizada por estudiantes de la Especialización en Seguridad y Salud en el Trabajo, previa comprensión del proyecto y capacitación mediante talleres sobre las características de los vendedores ambulantes y manejo expedito de la encuesta. Para la concentración de los vendedores se contó con el apoyo de la Beneficencia del Meta y de los distribuidoras de chance y lotería en Yopal, entidades que otorgaron tiempo para que los loteros y chanceros asistieran a la aplicación de la encuesta -en Villavicencio al local ubicado en el centro comercial Los Centauros y en Yopal al antiguo hospital-, y también se hizo búsqueda activa de los vendedores de lotería y chance en sus sitios de trabajo.

El análisis de los datos se realizó con el programa IBM SPSS Statistics Versión 22, previa codificación o asignación de números a los indicadores. Posteriormente se realizó análisis univariado y se describieron las variables mediante sus frecuencias absolutas y relativas y el cálculo de algunos estadísticos descriptivos como la moda, media, mediana y desviación estándar. Los sesgos de memoria en los accidentes de trabajo se controlaron ubicando eventos dentro de los últimos 12 meses.

En cuanto a las consideraciones éticas, se tuvo en cuenta la Resolución No. 08430 de 1993 (33), asegurando que es una investigación sin riesgo. Se obtuvo autorización y participación en el estudio de las organizaciones de los vendedores de lotería a través de oficio a las loterías y/o casas de chance que se enunciaron en el proyecto. El proyecto fue aprobado mediante el proceso de evaluación contemplado en la Convocatoria 2014 de la Dirección General de Investigaciones de la Universidad de los Llanos. Se solicitó la firma del consentimiento informado a todos los participantes en el estudio, garantizando la reserva del nombre y publicación de los datos en forma colectiva. Los autores afirman no tener conflicto de intereses.

\section{OBJETIVOS}

\section{Objetivo general}

Analizar las características sociodemográficas, percepción de la salud y riesgos ocupacionales de la población de vendedores de lotería de Villavicencio y Yopal.

\section{Objetivos específicos}

- Describir las características sociodemográficas y las condiciones de trabajo de la población de vendedores de lotería y chance de Villavicencio y Yopal.

- Establecer los factores ambientales que afectan a la población de vendedores de lotería y chance de Villavicencio y Yopal.

- Identificar la percepción de salud que presenta la población de vendedores de lotería y chance de Villavicencio y Yopal.

\section{RESULTADOS}

La recolección de la información se llevó a cabo entre los meses de septiembre y diciembre de 2015. La principal limitante estuvo constituida por la renuencia de los loteros de Villavicencio a participar, debido a incredulidad en torno a lo relacionado con acciones de atención a su problemática por parte del Gobierno.

\section{Características sociodemográficas, económicas y de seguridad social}

En cuanto al lugar de residencia, el 25,7\% de los participantes era de Yopal (Casanare) y 74,3\% de Villavicencio (Meta). La encuesta se ejecutó en la zona urbana; sin embargo, el 9,6\% era de la zona rural. Con respecto a la edad, la moda fue 62 años, media de 50,78 años, desviación estándar de 17,6 años, lo que implica que la población no fue homogénea. Según los parámetros anteriores, el $68 \%$ de la población se encuentra entre 33,18 - 68,38 años y el rango en donde se ubicó la mayoría de los vendedores estuvo entre 30-69 años. Es una población que, si bien está dentro de la población económicamente activa, también sobrepasa dicho rango con un $16,86 \%$ de 
mayores de 70 años todavía laborando. Sobre analfabetismo, el 7,2\%, no terminó su primaria, el $24,1 \%$ tiene la primaria incompleta, el $14,5 \%$ no término la secundaria, y solo el $15,2 \%$ del total de la muestra terminó estudios técnicos, tecnológicos y universitarios.

Acerca del género, la preponderancia observada de mujeres $(59,4 \%)$ en esta actividad de la economía informal se explica, en parte, por el reparto desigual del peso de las responsabilidades familiares no remuneradas, siendo la mujer en la que, por cultura, recaen dichas responsabilidades.

La tabla 1 muestra una población heterogénea en cuanto a etnia, con participación de un $22,5 \%$ entre indígenas, población afrocolombiana y raizal que, como grupos minoritarios, si bien gozan de políticas sociales y la normativa laboral formal no reconoce formas explícitas de discriminación o exclusión en Colombia, su capacidad de hacerlas valer está por evaluarse. El restante $77,5 \%$ evidenció que su informalidad no se relacionaba con condición de etnia sino que se debía a causas estructurales: en lo relacionado con violencia sociopolítica, un $9,2 \%$ era desplazado y un $0,4 \%$ desmovilizado.
ISSN 1794-9831

E-ISSN 2322-7028

Vol. 14 No. 1

Ene - Jun 2017

Cúcuta, Colombia 
Caracterización, riesgos ocupacionales y percepción de salud de vendedores informales de lotería y chance - Amalia Priscila PeñaPita, Martha Cecilia Sarmiento-Mejía, Ana Teresa Castro-Torres

ISSN 1794-9831

E-ISSN 2322-7028 Vol. 14 No. 1

Ene - Jun 2017

Cúcuta, Colombia
Tabla 1. Distribución porcentual de los vendedores de lotería y chance de Villavicencio y Yopal, según características sociodemográficas.

\begin{tabular}{|c|c|c|c|}
\hline \multicolumn{2}{|c|}{ Características sociodemográficas } & Frecuencia & Porcentaje \\
\hline Edad & $\begin{array}{l}<18 \\
18-29 \\
30-39 \\
40-49 \\
50-59 \\
60-69 \\
70-79 \\
80 y+\end{array}$ & $\begin{array}{c}4 \\
28 \\
44 \\
33 \\
35 \\
63 \\
34 \\
8\end{array}$ & $\begin{array}{c}1,61 \\
11,24 \\
17,67 \\
13,25 \\
14,06 \\
25,30 \\
13,65 \\
3,21\end{array}$ \\
\hline \multicolumn{2}{|r|}{ Total } & 249 & 100,0 \\
\hline \multirow{3}{*}{ Sexo } & Masculino & 101 & 40,6 \\
\hline & Femenino & 148 & 59,4 \\
\hline & Total & 249 & 100,0 \\
\hline \multirow{6}{*}{ Estado Civil } & Casado & 35 & 14,1 \\
\hline & Soltero & 90 & 36,1 \\
\hline & Viudo & 20 & 8,0 \\
\hline & Unión Libre & 75 & 30,1 \\
\hline & Separado & 29 & 11,6 \\
\hline & Total & 249 & 100,0 \\
\hline \multirow{3}{*}{ Zona de residencia } & Urbano & 225 & 90,4 \\
\hline & Rural & 24 & 9,6 \\
\hline & Total & 249 & 100,0 \\
\hline \multirow{9}{*}{ Nivel Escolaridad } & Ninguno & 18 & 7,2 \\
\hline & Primaria Incompleta & 61 & 24,5 \\
\hline & Primaria Completa & 47 & 18,9 \\
\hline & Secundaria Incompleta & 36 & 14,5 \\
\hline & Secundaria Completa & 49 & 19,7 \\
\hline & Técnico & 24 & 9,6 \\
\hline & Tecnológico & 3 & 1,2 \\
\hline & Universitario & 11 & 4,4 \\
\hline & Total & 249 & 100,0 \\
\hline \multirow{6}{*}{$\begin{array}{l}\text { Tipo de población a } \\
\text { que pertenece }\end{array}$} & Indígena & 2 & 0,8 \\
\hline & Afro Colombiano & 12 & 4,8 \\
\hline & Negro & 2 & 0,8 \\
\hline & Raizal & 40 & 16,1 \\
\hline & Otro & 193 & 77,5 \\
\hline & Total & 249 & 100,0 \\
\hline \multirow{4}{*}{$\begin{array}{l}\text { ¿En qué condiciones } \\
\text { se encuentra Ud. } \\
\text { actualmente? }\end{array}$} & Desplazado & 23 & 9,2 \\
\hline & Desmovilizado & 1 & 0,4 \\
\hline & NO Desplazado Ni Desmovilizado & 225 & 90,4 \\
\hline & Total & 249 & 100,0 \\
\hline $\begin{array}{l}\text { Motivo de } \\
\text { desplazamiento }\end{array}$ & $\begin{array}{l}\text { Violencia sociopolítica } \\
\text { Desastre natural } \\
\text { Otro } \\
\text { No aplica } \\
\text { Total }\end{array}$ & $\begin{array}{c}19 \\
2 \\
2 \\
228 \\
249\end{array}$ & $\begin{array}{c}7,6 \\
0,8 \\
0,8 \\
90,8 \\
100,0\end{array}$ \\
\hline
\end{tabular}

Fuente: Instrumento del Diagnóstico Nacional de condiciones de salud y trabajo de las personas ocupadas en el sector informal de la economía de 20 departamentos de Colombia y propuesta de monitoreo de estas condiciones, 2008. 
Según la figura 1, que contiene la distribución porcentual por régimen de seguridad social, del 100 $\%$ de los encuestados el $82,1 \%$ está afiliado a salud, el $26.6 \%$ al régimen contributivo y el $55,5 \%$ al régimen subsidiado; sin embargo, es altamente preocupante que un $17,9 \%$ no se encuentra afiliado, generando un alto riesgo o vulnerabilidad para dichos trabajadores, lo que redunda en el gasto de bolsillo familiar, que se ve afectado por el pago de los servicios e influye negativamente en el estado económico de la población. Por otro lado, un $20 \%$ es beneficiarios Se observa la responsabilidad estatal en la cobertura de la salud para la mayoría de la población estudiada y es de resaltar el porcentaje de personas que aún no se encuentra en ninguno de los dos regímenes, lo que incrementa su vulnerabilidad.

Lo anterior cobra gran importancia no sólo porque contar con afiliación al sistema de salud dignifica el trabajo sino porque el $6,25 \%$ del total de los encuestados manifestó haber sufrido, durante los últimos 12 meses, algún tipo de accidente grave relacionado con el trabajo, como por ejemplo fracturas o contusiones por accidentes de tránsito o caídas.

En lo relacionado con afiliación a pensiones, apenas se llega al 9,6\%, situación que, a corto y mediano plazo, dada la edad de la población estudiada, hace prever una situación alarmante, pues se generaría una gran dependencia de las personas que no aportan a pensión y que, en un futuro, van a depender totalmente del apoyo familiar, si lo tienen, o del Estado.

En lo atinente a la afiliación a la Administradora de Riesgos Laborales (ARL), la franja expuesta es una minoría (7,6\%), pero, en razón a desarrollar su trabajo de forma ambulante (en la calle), no está exenta de accidentarse y no posee cobertura de atención médica, rehabilitación y pago de prestaciones económicas como incapacidades y/o pensiones de invalidez, incrementando su vulnerabilidad.

Figura 1. Distribución porcentual de los vendedores de lotería y chance de Villavicencio y Yopal, según afiliación al Sistema de seguridad social $(n=249)$.

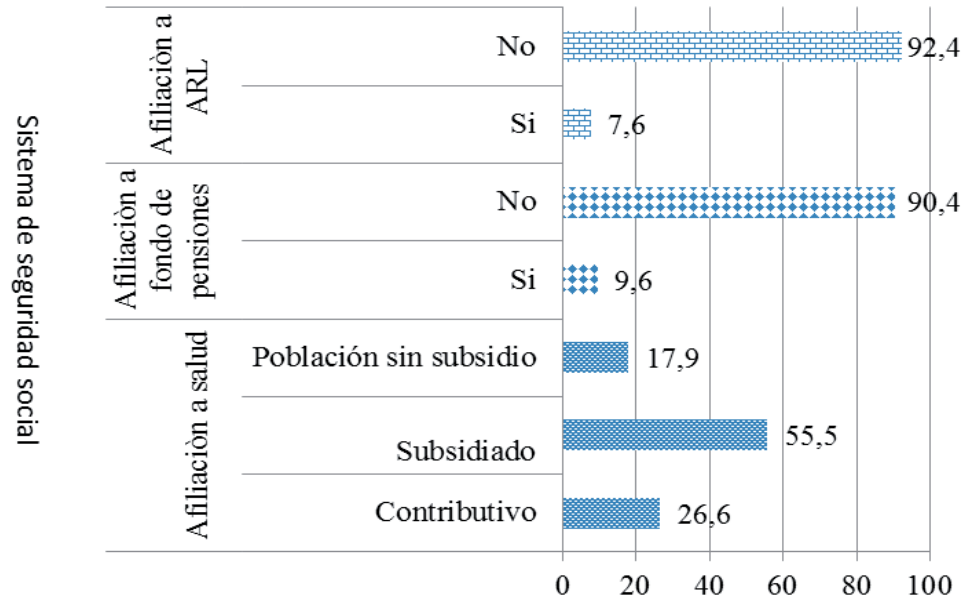

Porcentaje

Fuente: Instrumento del Diagnóstico Nacional de condiciones de salud y trabajo de las personas ocupadas en el sector informal de la economía de 20 departamentos de Colombia y propuesta de monitoreo de estas condiciones, 2008 . 
ISSN 1794-9831

E-ISSN 2322-7028

Vol. 14 No. 1

Ene - Jun 2017

Cúcuta, Colombia
Acerca del acceso a los servicios públicos domiciliarios, de los participantes en el estudio, dispone de energía el 79,5\%, de gas el $68,3 \%$, de acueducto el $79,5 \%$, de alcantarillado el $77,9 \%$, y tiene celular o teléfono fijo un $77,5 \%$. Se puede afirmar que las necesidades de servicios básicos de vivienda están cubiertas en tres cuartas partes de la población estudiada.

En relación con las condiciones socioeconómicas, los ingresos semanales tienen una media de $\$ 170.775$ que, calculado para el mes, alcanzaría los $\$ 683.100$ si los ingresos semanales fueran constantes, hecho que no ocurre en una actividad en donde el ingreso depende de la capacidad de la persona y de muchos otros factores. Los trabajadores informales de este sector devengan $\$ 581.532$ mensuales, resultado de $\$ 145.383$ semanales que, además son cifras menores al salario mínimo legal vigente (SMLV) para el 2015 (\$642.488) (34), lo que permite ubicar al $68 \%$ de la población con un ingreso semanal entre \$25.392 y $\$ 31.158$. Únicamente el 4,4 \% es propietario de una venta ambulante de productos varios (que incluye la venta de lotería y chance), es decir, trabaja por cuenta propia.

La figura 2 evidencia que la quinta parte de la población es pobre (58\%) y, además, el 20,1\% no está clasificada en el Sistema de Potenciales Beneficiarios para Programas Sociales (SISBEN), lo que los excluye de ser beneficiados en programas para población de escasos recursos, necesarios para la mayoría de ellos, puesto que viven en arriendo y tienen la responsabilidad económica de familia y son cabezas de hogar. Se puede afirmar, sin lugar a dudas, que es una población con alta vulnerabilidad.

Figura 2. Distribución porcentual de los vendedores de lotería y chance de Villavicencio y Yopal, según condiciones socioeconómicas $(n=249)$.

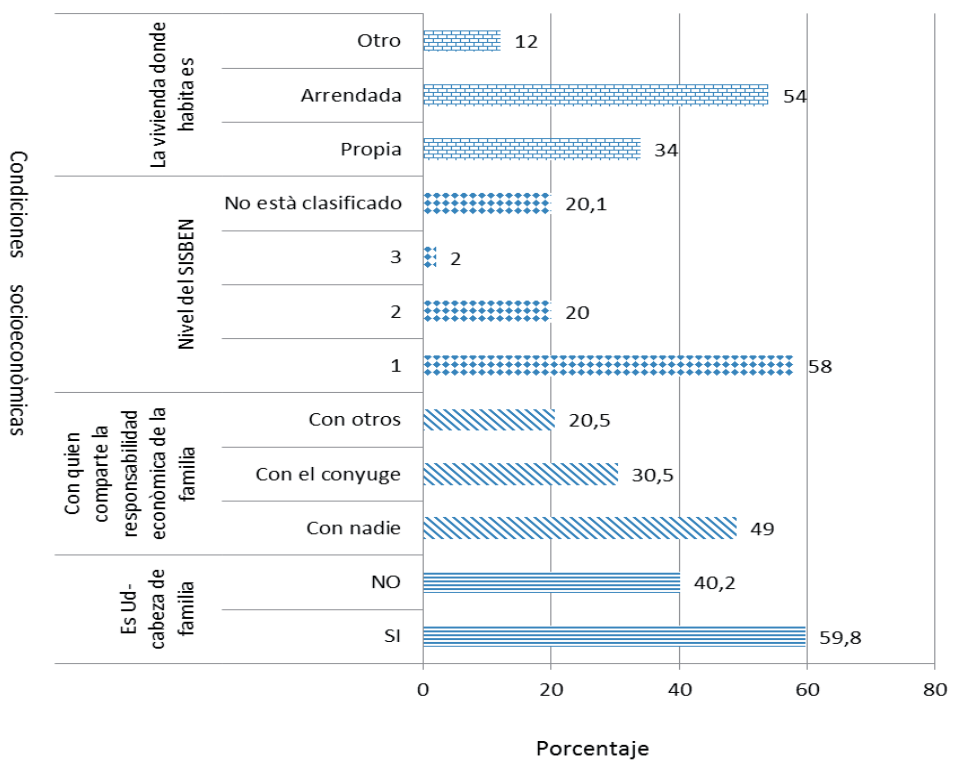

Fuente: Instrumento del Diagnóstico Nacional de condiciones de salud y trabajo de las personas ocupadas en el sector informal de la economía de 20 departamentos de Colombia y propuesta de monitoreo de estas condiciones, 2008. 


\section{Condiciones de trabajo}

$\mathrm{Al}$ respecto de la duración de la jornada laboral, si bien en promedio trabajan 8,95 horas, con una desviación estándar de 2,23 horas, se encontró que el $68 \%$ de la población de vendedores de lotería y chance labora un total de 6,7-11,2 horas diariamente. Sin embargo, existe un porcentaje representativo que trabaja entre 9 y 10 horas $(28,1 \%)$ y de 11 a 14 horas diarias $(17,1$ $\%)$. De igual forma, el $62,5 \%$ labora todos los días de la semana y, a pesar de ello, no logra cubrir sus necesidades básicas debido a los bajos ingresos que logra percibir como producto de su actividad. Ahora bien, la actividad exige trabajar en jornada nocturna a un $41,8 \%$ de los participantes en el estudio, quienes justificaron esta acción como tiempo necesario para completar el diario, situación que dificulta aún más el acceso a los servicios sanitarios, pues los locales en esa jornada ya están cerrados, además del incremento del riesgo a ser víctimas de robo de lo producido en el día.

Se encontró coherencia en los resultados al indagar sobre las relaciones interpersonales y resolución de conflictos en el trabajo, además de participación en riñas o peleas en horas de trabajo, descubriendo que un $71,1 \%$ conversa y resuelve problemas fácilmente con sus compañeros y únicamente un $10,4 \%$ se ha visto involucrado en riñas. Un aspecto que contribuye a este buen comportamiento tiene que ver con que sus puestos de trabajo gozan de espacio suficiente para moverse fácilmente ( $74 \%$ de los vendedores), lo que implica no incomodar al otro en su actividad.

\section{Factores ambientales}

Para el estudio de estos factores se observó el puesto de trabajo y se consideró como el espacio físico donde se realiza la actividad laboral (lugar de trabajo) en los estacionarios. Así, el vendedor ambulante, que en la muestra correspondió al $48,6 \%$, es quien está directamente más expuesto a factores ambientales peligrosos en su lugar de trabajo, en particular a sustancias químicas peligrosas suspendidas en el aire, radiaciones ionizantes y no ionizantes, rayos ultravioleta, ruido, vibraciones, altas y bajas temperaturas y humedad, entre otros factores. Indirectamente, todos los vendedores -estacionarios y semiestacionarios- están expuestos, puesto que laboran en el espacio público (acera) y, por lo tanto, les son aplicables las recomendaciones prácticas en el ambiente, de seguridad y salud en el trabajo establecidas por la Organización Internacional del Trabajo (35).

Adicionalmente, se encontró que, en el lugar de trabajo, los loteros y chanceros están expuestos igualmente a frío o calor exagerado y radiación solar en un $58,2 \%$ (sólo un $43,4 \%$ usa gorra, que deja expuesto el pabellón auricular y gran parte de la nariz) y a la lluvia el 56,6\%. También siente olor a humedad o recibe malos olores un $41,4 \%$, pero únicamente el $4,8 \%$ usa mascarilla.

Así mismo, los loteros y chanceros presentan exposición a productos químicos cuando se desplazan por algunas zonas donde se realizan actividades industriales y de latonería y pintura, entre otras actividades. Tal exposición se ha relacionado con enfermedades de vías respiratorias superiores, dermatológicas, cardiovasculares y del sistema periférico y nervioso central, anemia y enfermedades visuales. Otras causas de preocupación son el agotamiento y el shock térmico. Para ellos, el ambiente favorable, entorno en donde labora, lo configura el municipio de origen, dado que son ambulantes en su mayoría.

\section{Riesgos de ruido}

El tipo de venta expone a los vendedores a un ruido tan fuerte que les induce $(63,5 \%)$ a hablar en un tono más alto para la conversación con otros. La mayor fuente generadora de ruido la constituyen los vehículos $(79,9 \%)$ y se evidencia que el ruido es uno de los grandes problemas de los loteros y chanceros, puesto que lo padecen durante 7,23 horas de su jornada laboral, lo que implica que el $68 \%$ de esta población soporta ruido entre 3,73 a 10,73 horas diarias.

\section{Riesgos sanitarios}

Con respecto a las condiciones de higiene y limpieza en el lugar de trabajo, el $72,3 \%$ posee malas condiciones; únicamente tiene facilidad de acceso al servicio sanitario el 55\%. La recolección pública de basuras se efectúa cada dos días en el 90,4\% de los lugares de trabajo y el 32,1\% afirma que labora cerca de aguas estancadas, potenciales reservorios de vectores.

\section{Riesgos sociales}

Con respecto de la seguridad en su puesto de trabajo, un $81,5 \%$ de los vendedores de lotería y/o chance
ISSN 1794-9831

E-ISSN 2322-7028

Vol. 14 No. 1

Ene - Jun 2017

Cúcuta, Colombia 
ISSN 1794-9831

E-ISSN 2322-7028

Vol. 14 No. 1

Ene - Jun 2017

Cúcuta, Colombia afirma sentirse poco seguros $(43,4 \%)$ y muy inseguros $(38,1 \%)$, y para el $69 \%$ de ellos el motivo de mayor inseguridad lo constituye el robo. Si bien las mujeres son más propensas a ser víctimas de robos, analizando detalladamente la proporción de casos de esta índole y número de representantes de cada género, no se encontró diferencia significativa entre los dos géneros respecto del robo: tanto hombres como mujeres sufren por igual de la delincuencia común en un $30 \%$.

También se pudo apreciar que el $51 \%$ de la población se siente a gusto desempeñando la labor de venta de lotería y chance, pero las mujeres valoran esta tarea un $5 \%$ más que los hombres. Lo anterior se constituye en un factor que influye positivamente en el ánimo del trabajador informal, motivándolo a llevar a cabo su actividad aún en condiciones ambientales adversas (clima). De las personas inconformes con la labor que desempeñan, el $8.1 \%$ expresa que si tuvieran la oportunidad de cambiar de empleo lo harían.

Para el vendedor de lotería y chance el entorno en donde labora lo configura el municipio, dado que son ambulantes en su mayoria.

\section{Percepción de salud}

Como se puede observar en la figura 3 , la mayoría $(45,4 \%)$ de la población de vendedores de lotería y chance consideró regular o mala su salud. Este resultado se asocia con la percepción sobre los problemas de salud, fuertemente mediada por las condiciones materiales de vida y por la valoración de la salud en un contexto sociocultural específico, que como se muestra en la caracterización, las condiciones de la población de estudio son precarias.

Figura 3. Distribución porcentual de la población de estudio, según percepción de la salud.

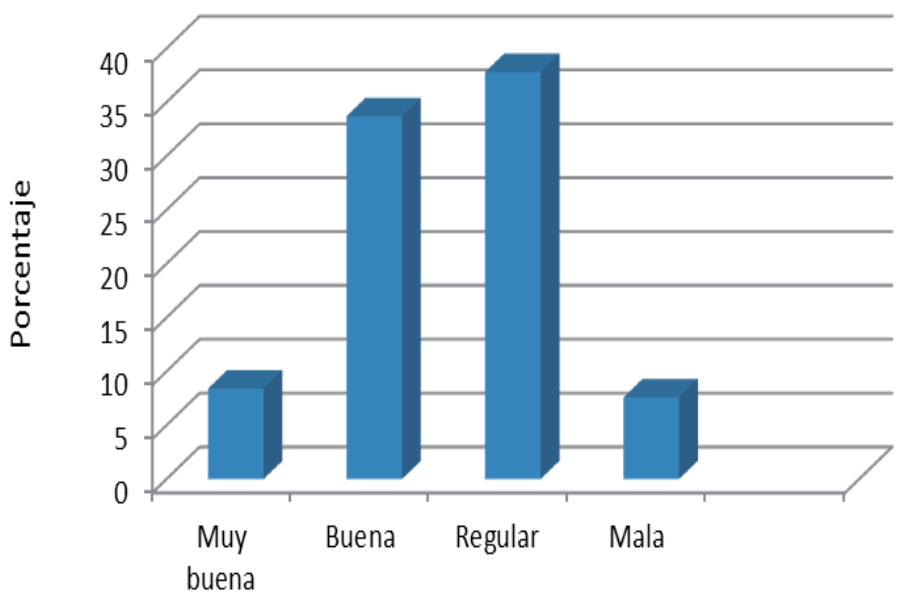

Autopercepción de salud

Fuente: Encuesta Nacional de Condiciones de Salud y Trabajo del Sector del Comercio Informal. Anexo III, 2008. p. 121.

\section{DISCUSIÓN}

Para la discusión se consideró el Diagnóstico nacional de condiciones de salud y trabajo de las personas ocupadas en el sector informal de la economía de 20 departamentos de Colombia (31), en el que se incluyeron loteros. Se carece de estudios específicos de vendedores de lotería y chance en el país, que sirvan de referente. Desde la salud pública, las condiciones de trabajo y salud de los vendedores informales revisten especial importancia en relación con la vulnerabilidad tanto social como ante exposición a riesgos y sin cobertura de protección frente a los mismos. 
En lo relacionado con la participación de la mujer en el oficio de vender de lotería y chance, fue superior 28,4 puntos porcentuales con respecto a lo reportado por el Ministerio de la Protección Social (31\%) (31), siendo así una relación mujer - hombre de 1,5:1 respectivamente. Este dato es similar a lo hallado por Galvis y Pérez (36), en la Región Pacífica, en donde la informalidad para el grupo de las mujeres es sistemáticamente más alta, lo que claramente indica una desventaja para ellas en el mercado de trabajo y, además, reafirma que la participación de la mujer se incrementa en el sector de servicios y en las actividades no reguladas (37).

Al respecto, en México (16), la participación de la mujer varía entre 20 y $30 \%$, cifra inferior a la hallada en el presente estudio, siendo mayor la participación del hombre como trabajador informal, lo cual corrobora que las mujeres tienden a ubicarse en el extremo inferior del abanico de ocupaciones en el sector informal, trabajando por cuenta propia o sin remuneración (38).

Dentro de la sociedad, en general, el hecho de que, tradicionalmente, las mujeres dediquen más horas para la realización de actividades no remuneradas que los hombres, como por ejemplo las labores domésticas, reduce la oportunidad de su participación en el mercado del trabajo y, si lo hace, las condiciones laborales le dificultan cumplir con su rol trabajador y percibir un salario. La presencia concentrada de la mujer en la informalidad, que es reconocida por el Ministerio del Trabajo, es impulsada por la independencia en el manejo del tiempo, en razón a que la mayoría son cabeza de familia (13).

Acerca de la edad, en el estudio de Rodríguez y Calderón (39), el rango en el que se ubicó la mayoría de la población fue 32-40 años, mientras que en el presente estudio se prolongó hasta edades mayores y estuvo entre 30-69 años. Llama la atención un grupo significativo de vendedores de lotería y chance $(16,86$ $\%$ ) que tiene 70 años y más, fenómeno explicado por el acceso limitado a otros oficios debido a la edad, como mencionan ellos mismos, y que se ubica en el grupo que el Banco Mundial (40) clasifica como por exclusión o involuntarios.

Con relación al nivel educativo, el analfabetismo es inferior en los loteros y chanceros en $3,12 \%$ con respecto al diagnóstico nacional (31), pero en la primaria incompleta, primaria completa y secundaria incompleta los demás niveles educativos superan a los loteros en $6,27 \%, 7,71 \%$ y $4,46 \%$ respectivamente. En los técnicos, tecnólogos y universitarios es superior la presencia de los loteros con $8,09 \%, 1,2 \%$ y $3,88 \%$ respecto al diagnóstico nacional (31), lo cual llama la atención debido a que podrían ubicarse en un oficio acorde con la formación y no es así. Esto podría estar reflejando la progresiva erosión de la capacidad de absorber empleo del sector formal manufacturero, que ha aumentado el desempleo y ha nutrido al sector informal urbano, el cual ha ido absorbiendo la fuerza de trabajo residual (41).

Al respecto, Garganta y Gasparini (42) mencionan que los trabajadores eligen óptimamente el sector donde ubicarse de acuerdo a su dotación de capital humano, esto es, que la ubicación laboral es dependiente no sólo de la cantidad sino también de la calidad, del grado de formación y de la productividad de las personas involucradas en un proceso productivo.

En lo atinente al régimen de afiliación, en el presente estudio se encontraron diferencias sustanciales, siendo mayor en un $21,6 \%$ los contributivos, inferior en un $13 \%$ los subsidiados y $10,7 \%$ de población pobre sin subsidio, con relación a los resultados del diagnóstico nacional (31), en donde el 5\% fue contributivo, el $68 \%$ subsidiado y el $27 \%$ vinculados. En cuanto a pensiones, la situación en general es crítica; sin embargo, los loteros y chanceros están $7,6 \%$ por encima de los informales reportados por el diagnóstico nacional (31), en donde la afiliación a pensión alcanzó únicamente el $2 \%$, lo cual puede estar relacionado, en Villavicencio, con el inicio de la política de formalización de los chanceros por parte de una de las empresas de chance y lotería que participó en el estudio.

Los resultados de la variable ingresos son contrarios al estudio de Rodríguez y Calderón (39), donde la mayoría de vendedores informales recibían entre uno y dos salarios mínimos legales vigentes, es decir, entre $\$ 566.700$ y $\$ 1.133 .400$, mientras que en el presente estudio la gran mayoría $(80 \%)$ de los vendedores de lotería y chance no logra devengar un SMLV. A lo anterior se le adiciona el hecho de ser cabeza de familia, situación que afecta la calidad de vida frente a las demandas de necesidades básicas y explica la ubicación de estos trabajadores en la línea de pobreza. De otro lado, en Argentina, con la mayoría
ISSN 1794-9831

E-ISSN 2322-7028

Vol. 14 No. 1

Ene - Jun 2017

Cúcuta, Colombia 
ISSN 1794-9831

E-ISSN 2322-7028

Vol. 14 No. 1

Ene - Jun 2017

Cúcuta, Colombia de población trabajadora en constante lucha contra la pobreza y la incertidumbre laboral, esta situación se refleja en la desigualdad alrededor de la distribución del ingreso, repercutiendo en la riqueza del país (39).

En cuanto a las formas de tenencia de la vivienda, estas contribuyen a deteriorar el presupuesto de los trabajadores, puesto que, tanto en el diagnóstico nacional (31) como en el presente estudio, la mayoría paga arriendo. Acerca de las otras formas de tenencia, la diferencia es superior un $8,2 \%$ en los vendedores de lotería y chance.

En lo relacionado con servicios básicos, el diagnóstico nacional (31) muestra un 10\% más de cobertura en energía y $3 \%$ en acueducto que el de vendedores de lotería y chance, situación que contrasta con la baja cobertura en alcantarillado y telefonía, en donde es de resaltar los 28,47 puntos porcentuales por encima de los datos del diagnóstico nacional (31), en cuanto a cobertura del alcantarillado de este estudio, e igualmente el acceso a teléfono, donde los resultados están por encima en 49,1 puntos, sobre los del diagnóstico nacional (31).

Cabe traer a discusión la necesidad de un acercamiento socio-ecológico, es decir, desde una visión holística, indispensable para generar y mantener ambientes favorables o propicios para la salud en población informal altamente vulnerable, como lo es la promoción de la salud, para lo cual el Ministerio de Salud (43) consideró la dimensión de la salud y el ámbito laboral con el fin de intervenir y propender por la salud y la seguridad en el trabajo.

Con respecto a la APES, en Argentina, el estudio de González et al. (44) determinó que está influenciada por el nivel de ingreso y la forma como dicho ingreso está distribuido en la sociedad: las personas con menor ingreso o en comunidades de mayor desigualdad tienden a percibir su estado de salud peor que aquellos con ingresos superiores o que están en comunidades más equitativas.

Sin embargo, De Maio (45), en Argentina, utilizó el coeficiente de correlación bivariante de Pearson $(0,09)$ y no encontró significancia estadística $(\mathrm{P}=0$, 05) entre ingreso familiar promedio y APES, pero sí con la interrupción de la actividad habitual $(0,71)$; lo que explica que el $45,4 \%$ de los loteros y chanceros perciban entre regular y mala su salud, puesto que los ingresos dependen en gran medida de la habilidad para lograr vender una cantidad de lotería o chance que les asegure un ingreso para su sustento, por lo que en una mala temporada, no alcanzan a percibir ni siquiera lo correspondiente a la fracción semanal de un SMLV.

En Colombia, para el mercado laboral de la economía informal es necesario considerar lo atinente a la calidad, que está relacionada con los ocupados en vender lotería o chance, empleos que son de baja productividad, con escaso capital físico y/o humano en los que no se reconoce, a la mayoría, las prestaciones sociales o en los que se incumple con las jornadas laborales. La situación anteriormente descrita, para los vendedores de lotería y chance del estudio, es similar a lo que ocurre en Ecuador (46), en donde la no afiliación de trabajadores, igualmente informales, es superior al $70 \%$.

\section{CONCLUSIONES}

Los vendedores de lotería y chance se encuentran en edad media de 50,78 años, la población en su mayoría está conformada por adultos mayores y mujeres, solteros o en unión libre, con primaria completa o incompleta, sus ingresos son menores de un salario mínimo legal mensual vigente y más de la mitad son cabeza de familia.

Las condiciones de trabajo son precarias, pues realizan jornadas de trabajo de entre 11 y 14 horas diarias, incluyendo horario nocturno. La mayoría trabaja todos los días de la semana, la afiliación al Sistema General de Seguridad Social en Salud cubre -a través del régimen subsidiado- a la mayoría de los trabajadores, carecen de afiliación en salud casi la quinta parte de los entrevistados y existe un bajo nivel de acceso a pensiones y riesgos laborales, con las implicaciones que esto conlleva en el futuro para la calidad de vida de ellos y sus familias.

Para los vendedores de lotería y chance se encontró que todos, directa e indirectamente, están expuestos a condiciones ambientales adversas por laborar en el espacio público; en razón a lo anterior, los factores de riesgo ocupacional prioritarios para ser intervenidos son: factores de riesgo climático, radiación solar, ruido, riesgos sanitarios y aquellos relacionados con la seguridad, lo cual conlleva estrés laboral derivado del riesgo de robo, que es su mayor amenaza. En lo que respecta a la autopercepción de salud, la mayoría la consideran entre buena y excelente. 
Esta es una población que requiere de intervenciones intersectoriales, dada la complejidad de su condición y la escasa capacidad organizativa para reivindicar sus derechos frente a la salud.

ISSN 1794-9831

E-ISSN 2322-7028

Vol. 14 No. 1

Ene - Jun 2017

Cúcuta, Colombia

\section{CONFLICTO DE INTERESES}

Las autoras declaran no tener ningún conflicto de intereses. 
ISSN 1794-9831

E-ISSN 2322-7028 Vol. 14 No. 1

Ene - Jun 2017 Cúcuta, Colombia

\section{REFERENCIAS BIBLIOGRÁFICAS}

1. Jiménez-Restrepo DM. La informalidad laboral en América Latina: ¿Explicación estructuralista o institucionalista? Cuadernos de Economía. Cuadernos de Economía. [Internet]. 2011 [consultado 15 de febrero de 2016]; 31(58):113-143. Disponible en: http://www.redalyc.org/pdf/2821/282125048006.pdf

2. República de Colombia, Departamento Administrativo Nacional de Estadística (DANE). Metodología informalidad gran encuesta integrada de hogares - GEIH. Censo [Internet]. 2009 [consultado 12 de agosto de 2016]. Disponible en: http://www.dane.gov.co/files/investigaciones/boletines/ech/ech informalidad/ metodologia informalidad.pdf

3. República de Colombia, Departamento Administrativo Nacional de Estadística (DANE). Medición del empleo informal por tamaño de establecimiento. Boletín técnico. [Internet]. 2016 [consultado 5 de octubre de 2016]. Disponible en: http://www.dane.gov.co/files/investigaciones/boletines/ech/ech informalidad/bol_ech informalidad_abr_jun16.pdf

4. Oficina Internacional del Trabajo (OIT). En América Latina y el Caribe al menos 127 millones de personas trabajan en la informalidad. Sala de prensa. [Internet]. Noticia 27 de agosto de 2013 [consultado 8 de febrero de 2016]. Disponible en: http://www.ilo.org/americas/sala-de-prensa/WCMS_220324/lang--es/index.htm,

5. Galvis LA. Informalidad laboral en las áreas urbanas de Colombia. Coyuntura Económica: investigación económica y social. [Internet]. 2012 [consultado 9 de febrero de 2016]; 42(1): 15-51 Disponible en: http://www. repository.fedesarrollo.org.co/bitstream/11445/271/2/Co_Eco_Sem1_2012_Galvis.pdf,

6. Naciones Unidas (UN), Comisión Económica para América Latina y el Caribe (CEPAL). División de Desarrollo Económico. Informalidad y tributación en América Latina: explorando los nexos para mejorar la equidad. [Internet]. 2012 [consultado 20 de marzo de 2016]. Disponible en: http://www.cepal.org/es/publicaciones/5356informalidad-y-tributacion-en-america-latina-explorando-los-nexos-para-mejorar-la

7. Salazar-Villamaría CI, Viveros- Aguilar JR. Condiciones de salud y trabajo de la población informal que labora en las galerías del municipio de Popayán, Colombia. Revista Cubana de Salud y Trabajo [Internet]. 2013 [consultado 22 de febrero de 2016]; 14(3): 11-23. Disponible en: http://bvs.sld.cu/revistas/rst/vol14_3_ 13/rst02313.htm

8. Rincón-Báez WU, Soler-Hurtado AJ. Perspectivas socioeconómicas de los vendedores informales de Chapinero, en Bogotá, Colombia. Cooperativismo y desarrollo [Internet]. 2015 [consultado 12 de abril de 2016]:24 (107). Disponible en: http://revistas.ucc.edu.co/index.php/co/article/view/1255

9. Senado de la República de Colombia. Informe consolidado al proyecto de Ley 138 de 2015 Senado, 200 de 2015 Cámara, febrero 24, por la cual se expide el Plan Nacional de Desarrollo 2014-2018: todos por un nuevo país. [Internet]. Bogotá D.C.: Senado de la República de Colombia; 2015 [consultado 11 de junio de

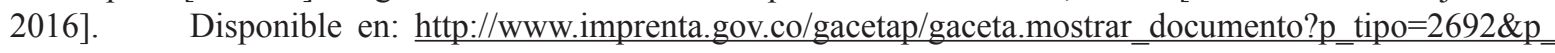
numero $=138 \& p \_$consec $=41287$

10. Diego Segura B, Guimaraens Juanena D, Rupérez Calvo MJ. Sol en el trabajo, un peligro olvidado. Seguridad y Salud en el Trabajo. [Internet]. 2010 [consultado 4 noviembre de 2016]; 57: 13-14. Disponible en: https://www. fundacionmapfre.org/documentacion/publico/i18n/catalogo_imagenes/grupo.cmd?path=1060058

11. United States of America. Department of Labor. Administración de Seguridad y Salud Ocupacional. Agua, sombra y descansos. Sin ellos no se puede trabajar. OSHA [Internet]. 2014 [consultado 1 noviembre de 2016]. Disponible en: https://www.osha.gov/SLTC/heatillness/spanish/index_sp.html

12. Blandón-Mena M. El trabajo en las calles: territorialización, control y política en el centro de Medellín a comienzos del siglo XXI. [Tesis magister en internet]. Medellín, Colombia: Universidad de Antioquia; 2011 [consultado 4 de noviembre de 2016]. 145 p. Disponible en: http://bibliotecadigital.udea.edu.co/bitstream/10495/1509/1/EL\%20 TRABAJO $\% 20$ EN\%20LAS\%20CALLES\%20TERRITORIALIZACI $\%$ C $3 \% 93 \mathrm{~N}, \% 20$ CONTROL $\% 20 \mathrm{Y} \% 20$ POL\%C3\%8DTICA\%20EN\%20EL\%20CENTRO\%20DE\%20MEDELL\%C3\%8DN\%20A\%20COMIEN.pdf

13. Ministerio del Trabajo de Colombia. Programa nacional de equidad laboral con enfoque diferencial de género. [Internet]. Bogotá D.C: Mintrabajo; 2013 [consultado 20 de agosto de 2016]. Disponible en: http://www. mintrabajo.gov.co/equidad/enfoque-de-genero

14. Mossey JM, Shapiro E. Self-rated health: a predictor of mortality among the elderly. Am J Public Health. [Internet]. 1982 [consultado 26 de marzo de 2016]; 72(8):800-808. Disponible en: http://www.ncbi.nlm.nih.gov/pubmed/7091475

15. Gómez-Montes JI, Curcio-Borro CL. Autopercepción de salud, presencia de enfermedades y discapacidades en ancianos de Manizales. Rev Asoc. Colomb. Gerontol. Geriatr. [Internet]. 2004 [consultado 22 de marzo de 2016]; 18 (4): 706-715. Disponible en: http://www.acgg.org.co/descargas/revista-18-4.pdf

16. Guadarrama R, Hualde A, López $\mathrm{S}$ (coordinadores). La precariedad laboral en México: Dimensiones, dinámicas y significados. [Internet]. México: El Colegio de la Frontera Norte/ Universidad Autónoma Metropolitana- 
Unidad Cuajimalpa; 2015 [consultado 12 de agosto de 2016. p. 42, 46. Disponible en: http://libreria.colef.mx/ detalle.aspx?id $=7492$

17. Organización Internacional del Trabajo (OIT). Promover el empleo, proteger a las personas. Economía informal en Argentina. [Internet]. 2011 [consultado 17 de mayo de 2016]. Disponible en: http://www.ilo.org/buenosaires/ temas/economia-informal/lang--es/index.htm

18. Cassirer N, Addati L. Ampliar las oportunidades de trabajo de la mujer: los trabajadores de la economía informal y la necesidad de servicios de cuidado infantil. Oficina Internacional del trabajo. [Internet]. 2007 [consultado 16 de mayo de 2016]. 18 p. Disponible en: http://www.ilo.org/wcmsp5/groups/public/---ed_protect/---protrav/--travail/documents/publication/wcms_145655.pdf

19. Figueroa-Socarías CA. Determinantes de la informalidad laboral y el subempleo en las áreas metropolitanas de Barranquilla, Cartagena y Montería. [Internet]. Bogotá D.C.: Ediciones Uninorte; 2010 [consultado 10 de abril de 2016]; 32:10-30. Disponible en: https://guayacan.uninorte.edu.co/publicaciones/upload/File/Documentos IEEC 32.pdf,

20. Mondragón-Vélez C, Peña X, Wills D. Labor Market Rigidities and Informality in Colombia. Documentos CEDE, Universidad de los Andes. [Internet]. 2010 [consultado 10 de febrero de 2016]; p. 3-22. Disponible en: http://www.cid.harvard.edu/Economia/papers\%20issues/Fall\%202010/Labor_Market_Rigidities_and Informality $07 \quad 102010 . p d f$

21. Bustamante-Izquierdo JP. Los retos de la economía informal en Colombia. Notas Fiscales [Internet]. 2011 [consultado 3 de febrero de 2016]; (9): 1-31. Disponible en: http://www2.congreso.gob.pe/sicr/cendocbib/con4 uibd.nsf/F92EE46AD221CB7605257D7A0074FC80/\$FILE/RetosEconomiaInformalColombia.pdf

22. Guataquí JC, García AF, Rodríguez M. El perfil de la informalidad laboral en Colombia. Perfil de Coyuntura Económica. [Internet]. 2011 [consultado 20 de febrero de 2016]; (16): 91-115. Disponible en: https:// aprendeenlinea.udea.edu.co/revistas/index.php/coyuntura/article/view/9629/8869

23. Bernal R. The Informal Labor Market in Colombia: identification and characterization. Desarrollo y Sociedad [Internet]. 2009 [consultado 11 de agosto de 2016]. (Primer semestre): 145-208. Disponible en: http://www. scielo.org.co/pdf/dys/n63/n63a05.pdf-

24. Ardila-Jaimes $C$, Rodríguez-Amaya RM. Condiciones de salud y laborales de la población trabajadora informal en situación de desplazamiento de Bucaramanga, Colombia. Investigación Andina. [Internet]. 2013 [consultado 22 de enero de 2016]; 26(15): 628-638. Disponible en: http://www.scielo.org.co/pdf/inan/v15n26/v15n26a02. pdf

25. Landínez E, Rojas H. Caracterización del comercio informal callejero en la ciudad de Bucaramanga. [Tesis en internet]. Bucaramanga: Universidad industrial de Santander: 2004 [consultado 20 de febrero de 2016]. Disponible en: http://repositorio.uis.edu.co/jspui/bitstream/123456789/8510/2/114346.pdf

26. Muñoz-Caicedo A, Chois-Lenis P. Riesgos laborales en trabajadores del sector informal del Cauca, Colombia. Rev.fac.med. [Internet]. 2014 [consultado 3 de febrero de 2016]; 62(3): 390-399. Disponible en: http://www. scielo.org.co/scielo.php?script=sci arttext\&pid $=\mathrm{S} 0120-00112014000300007 \& \operatorname{lng}=\mathrm{es} \& \mathrm{nrm}=$ iso $\mathrm{http}: / / \mathrm{dx} . \mathrm{doi}$. org/10.15446/revfacmed.v62n3.38682.

27. Secretaría Municipal de Salud de Villavicencio. Estudio de diagnóstico de la población de vendedores de lotería y chance. Documento Gris. Villavicencio: Secretaría Municipal de Salud; 2012.

28. Gobernación del Quindío, Universidad del Quindío. Caracterización de la población afrodescendiente en el departamento del Quindío. [Internet]. 2013 [consultado 16 de marzo de 2016]; 7-66 Disponible en: http:// quindio.gov.co/home/docs/items/item 193/INFORME_FINAL_ESTUDIO Y CARACTERIZACION_DE_ LA POBLACION_AFRODESCENDIENTE EN_EL DEPARTAMENTO DEL QUINDIO.pdf

29. Salinas JD, González SI, Marín LJ. Características de la Población Ocupada en Colombia: Un análisis del perfil de los formales e informales. Perfil de Coyuntura Económica. [Internet]. 2012 [consultado 18 de marzo de 2016]; (20): 57-86. Disponible en: http://aprendeenlinea.udea.edu.co/revistas/index.php/coyuntura/article/ view/18186/15635

30. Universidad de Nariño, Centro de Estudios en Salud. Caracterización de las condiciones de salud y laborales de trabajadores del sector informal del departamento de Nariño. [Internet]. 2010 [consultado 24 de febrero de 2016]; 10-30. Disponible en: http://idsn.gov.co/site/images/publicaciones/riesgos laborales/EL TAMBO/ UDENAR\%20CESUN\%20EL\%20TAMBO\%20.pdf

31. República de Colombia, Ministerio de la Protección Social. Diagnóstico Nacional de condiciones de salud y trabajo de las personas ocupadas en el sector informal de la economía de 20 departamentos de Colombia y propuesta de monitoreo de éstas condiciones. [Internet]. 2008 [consultado 24 de febrero de 2016]; 20-90 Disponible en: https:// www.minsalud.gov.co/sites/rid/Lists/BibliotecaDigital/RIDE/VP/DOA/diagnostico-nacional-de-condiciones-desalud-y-trabajo-de-las-personas-ocupadas-en-el-sector-informal-de-la-economia.pdf 
ISSN 1794-9831

E-ISSN 2322-7028 Vol. 14 No. 1 Ene - Jun 2017 Cúcuta, Colombia
32. Organización Panamericana de la Salud (OPS), Organización Mundial de la Salud (OMS). Estrategia de promoción de la salud en los lugares de trabajo de América Latina y el Caribe. Anexo No. 6: Estrategia de promoción de la salud en los lugares de trabajo de América Latina y El Caribe. Versión revisada, Marzo 20 de 2000. [Internet]. 2000 [consultado 23 de abril de 2016]; p. 4-8. Disponible en: http://www.who.int/occupational health/regions/en/oehpromocionsalud.pdf

33. Ministerio de Salud y Protección Social de Colombia. Resolución 08430 de 1993, 4 de octubre, por la cual se establecen las normas científicas, técnicas y administrativas para la investigación en salud. [Internet]. Bogotá D.C.: El Ministerio; 1993 [consultado 24 de marzo de 2016]. Disponible en: https://www.invima.gov.co/ images/pdf/medicamentos/resoluciones/etica res 8430 1993.pdf

34. Ministerio del Trabajo de Colombia. El salario mínimo subiría $\$ 26.488$ para el 2015. [Internet]. Jueves 4 de diciembre de 2014 [consultado 21 de agosto de 2016]. Disponible en: http://www.mintrabajo.gov.co/diciembre2014/4063-el-salario-minimo-subiria-26488-para-el-2015.html

35. Organización Internacional del Trabajo (OIT). Factores ambientales en el lugar de trabajo. Repertorio de recomendaciones prácticas en el ambiente de trabajo, seguridad en el trabajo, salud en el trabajo en Ginebra Suiza. [Internet]. Ginebra: OIT; 2001 [consultado 26 de marzo de 2016]; p. 24-65. Disponible en: http://www.ilo.org/ wcmsp5/groups/public/@ed_protect/@protrav/@safework/documents/normativeinstrument/wcms 112584.pdf

36. Gálvis-Aponte L, Pérez-Valbuena G. Informalidad laboral y calidad del empleo en la Región Pacífica colombiana. Documentos de Trabajo sobre Economía Regional. [Internet]. 2015 [consultado 26 de marzo de 2016]; (233): 1-50. Disponible en: http://www.banrep.gov.co/sites/default/files/publicaciones/archivos/dtser_233.pdf

37. Sanchis N, Katzkowicz N. Obstáculos para la inserción laboral equitativa y el empoderamiento económico de las mujeres. [Internet]. Argentina: Programa de la Naciones Unidas para el Desarrollo (PNUD), Agencia Española de Cooperación Internacional para el Desarrollo (AECID); 2014 [consultado 3 de abril de 2016]; p. 6-26 Disponible en: http://www.redetis.iipe.unesco.org/wp-content/uploads/2016/01/PNUD_ARGENTINA Obstaculos-p insercion laboral Equitativa.pdf

38. Álvarez-Vos OS. Informalidad laboral: Situación de las mujeres en Colombia. [Internet]. Bogotá D.C.: Universidad Tadeo Lozano; 2011 [consultado 8 de marzo de 2016]; p. 1-10. Disponible en: http://www. colombianistas.org/Portals/0/Congresos/Documentos/CongresoXVIII/Alvarez Oriana Sofia.pdf

39. Rodríguez-Lozano GI, Calderón-Díaz MA. La economía informal y el desempleo: el caso de la ciudad de Bucaramanga (Colombia). REV. INNOVAR. [Internet]. 2015 [consultado 21 de agosto de 2016]; 25(55): 41-58. Disponible en: http://www.fce.unal.edu.co/media/files/documentos/Innovar/v25n55/v25n55.pdf

40. Perry GE, Maloney WF, Arias Os, Fajnzylber P, Mason AD, Saavedra J. Informality Exit and Exclusion. World Bank Latin American And Caribbean Studies [Internet]. Washington D.C., USA: The World Bank; 2008 [consultado 6 de julio de 2016]; p. 5-6. Disponible en: http://www-wds.worldbank.org/external/default/WDSContentServer/WDSP/ IB/2007/06/19/000090341 20070619143652/Rendered/PDF/400080Informal101OFFICIAL0USE0ONLY1.pdf.

41. Cimoli M, Primi A, Pugno M. Un modelo de bajo crecimiento: La informalidad como restricción estructural. Revista de la Cepal No. 88. [Internet]. Abril 2006 [consultado 6 de julio de 2016]; p.89-107. Disponible en: http://archivo.cepal.org/pdfs/revistaCepal/Sp/088089107.pdf

42. Garganta S, Gasparini L. El Impacto de un Programa Social sobre la Informalidad Laboral: El Caso de la AUH en Argentina. [Tesis en internet]. Argentina: Universidad Nacional de la Plata; 2012 [consultado 22 de agosto de 2016]; p.7. Disponible en: http://cedlas.econo.unlp.edu.ar/archivos upload/doc cedlas133.pdf

43. República de Colombia, Ministerio de la Salud y Protección Social. Plan Decenal de Salud Pública. Dimensión salud y ámbito laboral. [Internet]. 2013 [consultado 21 de agosto de 2016]. Disponible en: https://www.minsalud. gov.co/plandecenal/Documents/dimensiones/dimension-salud-ambitolaboral.pdf

44. González JI, Sarmiento A, Alonso CE, Angulo R, Espinosa F. Efectos del nivel de ingreso y la desigualdad. Sobre la autopercepción en salud: Análisis para el caso de Bogotá. Revista Gerencia y Políticas de Salud. [Internet]. 2005 [consultado 17 de febrero de 2016]; 4(9): 120-140. Disponible en: http://www.redalyc.org/pdf/545/54540906.pdf

45. De Maio FG. Desigualdad en el ingreso como determinante social de la salud. SALUD COLECTIVA, Buenos Aires [Internet]. 2010 [consultado 22 de agosto de 2016]; 6(2): 195-209. Disponible en http://www.scielo.org. ar/pdf/sc/v6n2/v6n2a06.pdf

46. The World Bank. Ecuador the Faces of Informality. Report No. 67808-EC. [Internet]. Washington DC: The World Bank; 2012 [consultado 6 de julio de 2016]; p. 22. Disponible en: http://www-wds.worldbank.org/ external/default/WDSContentServer/WDSP/IB/2012/09/05/000356161_20120905032554/Rendered/PDF/6780 80ESW0P1260Faces0of0Informality.pdf 